\title{
Nrf1 is not a direct target gene of SREBP1, albeit both are integrated into the rapamycin- responsive regulatory network in human hepatoma cells
}

\author{
Keli Liu ${ }^{1}$, Shaofan $\mathrm{Hu}^{1}$, Lu Qiu ${ }^{1,2}$, Meng Wang ${ }^{1}$, Zhengwen Zhang ${ }^{3}$, and Yiguo Zhang ${ }^{1}$ \\ ${ }^{1}$ The Laboratory of Cell Biochemistry and Topogenetic Regulation, College of Bioengineering and Faculty of Medical Sciences, \\ Chongqing University, No. 174 Shazheng Street, Shapingba District, Chongqing 400044, China \\ ${ }^{2}$ School of Life Sciences, Zhengzhou University, No. 100 Kexue Avenue, Zhengzhou 450001, Henan, China \\ ${ }^{3}$ Laboratory of Neuroscience, Institute of Cognitive Neuroscience and School of Pharmacy, University College London, 29-39 \\ Brunswick Square, London WC1N 1AX, England, United Kingdom \\ * Correspondence: yiguozhang@cqu.edu.cn, or eaglezhang64@gmail.com
}

Abstract: It is worth interrogating why no more experimental evidence confirming those findings, since being reported by Manning's group in 2014's Nature (doi: 10.1038/nature13492), has been provided in the hitherto known literature. A key issue arising from their work is of particular concern about whether the mTORC1 signaling to upregulation of Nrf1-targeted proteasomal expression profiles occurs directly by SREBP1. In this study, our experiment evidence revealed that Nrf1 is not a direct target of SREBP1, although both are involved in the rapamycin-responsive regulatory networks. Closely scrutinizing two distinct transcriptomic datasets unraveled no significant changes in transcriptional expression of Nrf1 and almost all

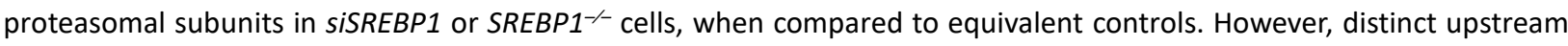
signaling to Nrf1 dislocation by p97 and its processing by DDI1/2, along with downstream proteasomal expression, may be monitored by mTOR signaling, to various certain extents, depending on distinct experimental settings in different types of cells. Our further evidence has been obtained from $D D I 1^{-1-}\left(D D / 2^{\text {ins }}\right)$ cells, demonstrating that putative effects of mTOR on the rapamycin-responsive signaling to Nrf1 and proteasomes may also be executed partially through a DDI1/2-independent mechanism, albeit the detailed regulatory events remain to be determined.

Key words: Nrf1, mTOR, SREBP1, p97/VCP, DDI1/2, proteasome, and transcriptional regulation.

\section{Introduction}

The normal homeostasis must have to be maintained in all healthy life forms, due to homeostasis robustness, plasticity and resilience ensuring that their structural organization, physiological function and biological behavior are being properly performed and perpetuated at a stable, robust steady-state (1). Conversely, defects in the maintenance of cell fitness and homeostasis have emerged as an underlying feature of a vast variety of pathologies, such as cancer, senescence and agingrelated diseases (2-4). Of note, protein homeostasis (i.e. proteostasis) is preserved or not, depending on a steady balance between protein synthesis and turnover $(5,6)$. Interestingly, as reported by Manning's group (7), such a finely-programmed balance between protein synthesis and degradation was coordinately regulated by the mechanistic target of rapamycin complex 1 (mTORC1), a central kinase that is generally activated by cell growth- and proliferation signaling to trigger protein translation (8). Besides, they had also shown that mTORC1 signaling activates transcriptional expression of nuclear factor erythroid 2-related factor 1 (Nrf1 with multiple isoforms, encoded by NFE2L1) directly by sterol regulatory element-binding protein 1 (SREBP1) $(7,9)$, although SREBP1 has been commonly accepted as a key control of lipid synthesis and membrane homeostasis, even in response to the rather multifaceted mTOR signaling (10-12).

However, a core point from Manning's work on the control of proteasomal proteolysis by mTOR (7) was first challenged by Goldberg's group, arguing that their methodology to measure the rates of protein degradation (labelled by ${ }^{35} \mathrm{~S}-\mathrm{Met} / \mathrm{Cys}$ rather than by ${ }^{3} \mathrm{H}$-Phe) and the former labelled-protein pulse chase experimental data appear questionable (13), because the resulting data were considered to be rather inconsistent with those well-established discoveries $(14,15)$. Amongst the best-studied actions of mTORC1 are enhancing protein synthesis, and also inhibiting protein degradation by autophagy and proteasomes $(14,16)$. While mTORC1 is inactivated during starvation, an increase in proteolysis and autophagy provides a recycle of amino acids for next protein synthesis and energy production. As such, Manning's group showed that inhibition of mTORC1 activity for $16 \mathrm{~h}$ or more resulted in a delayed reduction in overall proteolysis by downregulating transcriptional expression of proteasomal subunits (7). Similar results were also obtained from ${ }^{3} \mathrm{H}$-Phe-labelled pulse chase experiments in their reply to doubt by Goldberg's group (17). Such discrepant results presented by Manning's 
and Goldberg's groups are not attributable to nuances in the assays, but are inferable due to differences in their chosen culture conditions (17). A higher dose of rapamycin ( $300 \mathrm{nM}$, at least 100 -fold more than the $\mathrm{IC}_{50}$ for inhibiting mTORC1) was employed (13) to enable two mTOR kinase complexes (i.e., mTORC1 and mTORC2) to be completely blocked in mouse embryonic fibroblasts (MEFs) with genetic loss of tuberous sclerosis complex 2 (TSC2 ${ }^{-1}$, that is accompanied by aberrant activation of mTOR). By contrast, a much lower dose (20 nM) of rapamycin for treatment of $T S C 2^{-/}$cells grown in the lowserum conditions $(7,17)$ enabled for specific separation of effects of mTORC1 from mTORC2, as described by (18). Just under this status, it was found that $T S C 2^{--}$-leading activation of $\mathrm{mTORC1}$, rather than $\mathrm{mTORC2}$, stimulates a transcriptional programme involving SREBP1 and Nrf1, leading to an evident enhancement of proteasome-mediated proteolysis exclusively by Nrf1, but not Nrf2 $(7,9)$.

Since Manning's work was fantastically well done $(7,17)$ and has gained nearly 200 citations (from the Web of Science at https://www.webofscience.com), it is worth interrogating why no more further experimental evidence confirming their findings has been provided to date by any other groups, so far as we know. Of particular concern is a key issue arising from Manning's work, which merits reexamination of whether mTORC1 signaling to upregulation of Nrf1-targeted proteasomal expression profiles occurs directly by SREBP1, because this controversial mechanism remains obscure. Here, we found that transcriptional expression of Nrf1 and all proteasomal subunits is almost unaffected by SREBP1 (or SREBP2), but conversely, Nrf1 contributes to negative regulation of SREBP1 involved in lipid metabolism ((19) and this study). Besides, a Yin-Yang relationship between Nrf1 and SREBP2 for maintaining cholesterol homeostasis was elaborately unraveled in Hotamisligil's laboratory (20). Recently, a mechanistic study by Xu's group (21) has shown that activity of SREBPs is inhibited by promoting degradation of SREBP-cleavage activating protein (SCAP, a central sensor for cholesterol) through the protein-ubiquitin E3 ligase RNF5-dependent proteasomal pathway, in this process whereby this ligase is recruited to the endoplasmic reticulum (ER)-localized transmembrane protein 33 (TMEM33), a direct target of Nrf1. Therein TMEM33 is also a downstream effector of pyruvate kinase isoform 2 (PKM2), which coordinates together with p97/VCP to control the processing of Nrf1 and SREBPs, as well as their bidirectional regulatory ability to dictate lipid metabolism and homeostasis (21).

Collectively, the aforementioned findings appear to be together drawn into an argument against the central conclusion from the Manning's work. In the present study, our evidence has been presented revealing that Nrf1 is not a direct target of SREBP1, albeit they are involved in the rapamycin-responsive regulatory networks. Further experiments are designed to determine whether p97, DDI1 (also called VSM1 (v-SNARE binding protein-1), which is a highly conserved aspartyl protease among all eukaryotes from yeast to human (22-24) and DDI2 (only present in vertebrates (25) exert the putative rapamycinresponsive effects on Nrf1 processing and activity to regulate the proteasomal expression. Moreover, the human HepG2derived $D D / 1^{-1-}\left(D D / 2^{i n s}\right)$ cell line and tumor xenograft model have been established herein, so as to further elucidate the rapamycin-responsive effects on Nrf1 and cognate proteasomes.

\section{Materials and Methods}

\subsection{Cell culture and treatments}

HepG2 and HL7702 cell lines were grown in DMEM supplemented with $5 \mathrm{mM}$ glutamine, 10\% (v/v) foetal bovine serum (FBS), 100 units $/ \mathrm{ml}$ of either of penicillin and streptomycin, in a $37{ }^{\circ} \mathrm{C}$ incubator with $5 \% \mathrm{CO}_{2}$. Additional HepG2derived cell lines with knockout of $D D / 1^{-/}$were herein established by CRISPR-editing of DDI1 with specific gRNA (Table S1). The authenticity of $\mathrm{DDI}^{-/}$cells had been confirmed by its authentication analysis. Thereafter, experimental cells were transfected with a Lipofectamine 3000 mixture with indicated plasmids or siSREBP1 (with a pair of sequences, Table S1) for $8 \mathrm{~h}$, and allowed for a recovery from transfection in a fresh medium for $24 \mathrm{~h}$ before being experimented. Additional cells were treated with the mTOR inhibitor rapamycin (RAPA, 20 to $200 \mathrm{nM}$ ) or proteasomal inhibitor MG132 (1 to $10 \mu \mathrm{M})$ for different time periods $(4,16$ or $24 \mathrm{~h})$.

\subsection{Expression constructs}

An expression construct for human SREBP1 was made by cloning its full-length cDNA sequence into the pcDNA3 vector with a pair of its forward and reverse primers (Table S1), that were synthesized by Sangon Biotech Co. (Shanghai, China). 
Another expression plasmid of Nrf1 was reported previously (26). The fidelity of all these constructs was confirmed to be true by sequencing.

\subsection{Luciferase reporter assay}

After experimental cells $\left(1.0 \times 10^{5}\right)$ were allowed for growth in each well of the 12 -well plates to reach $80 \%$ confluence, they were co-transfected with a Lipofectamine 3000 mixture with pNrf1-luc or pNrf2-luc established by Qiu et al (27), plus other expression plasmids. In this dual reporter assay, the Renilla expression by pRL-TK served as an internal control for transfection efficiency. The resulting data were normalized from at least three independent experiments, each of which was performed in triplicate, and thus shown as a fold change (mean \pm S.D) relative to the control values.

\subsection{Quantitative real-time PCR}

About $500 \mathrm{ng}$ of total RNAs from experimental cells were subjected to reverse-transcriptase reaction to generate the first strand of cDNA. The newly-synthesized cDNA was used as the template for quantitative PCR in the Master Mix, before being deactivated at $95^{\circ} \mathrm{C}$ for $10 \mathrm{~min}$, and amplified by 40 reaction cycles of annealing at $95^{\circ} \mathrm{C}$ for $15 \mathrm{~s}$ and then extending at $60^{\circ} \mathrm{C}$ for $30 \mathrm{~s}$. The final melting curve was validated to examine the amplification quality. While $\beta$-actin mRNA level was employed as an optimal internal standard control, target gene expression levels were determined by quantitative real-time PCR, as described previously (28), with each pair of the indicated primers (Table S1). The resulting data were shown a fold change (mean \pm S.D) relative to the control values.

\subsection{Western blotting with distinct antibodies}

Total cell lysates in a lysis buffer (0.5\% SDS, $0.04 \mathrm{~mol} / \mathrm{L}$ DTT, pH 7.5) with protease and phosphatase inhibitors (each of cOmplete and PhosSTOP EASYpack tablets in $10 \mathrm{ml}$ buffer), were denatured immediately at $100^{\circ} \mathrm{C}$ for 10 min, sonicated sufficiently, and diluted in $3 \times$ loading buffer $(187.5 \mathrm{mmol} / \mathrm{L}$ Tris- $\mathrm{HCl}, \mathrm{pH}$ 6.8, 6\% SDS, 30\% Glycerol, $150 \mathrm{mmol} / \mathrm{L}$ DTT, 0.3\% Bromphenol Blue) at $100^{\circ} \mathrm{C}$ for $5 \mathrm{~min}$. Subsequently, equal amounts of protein extracts were subjected to separation by SDS-PAGE containing 4-15\% polyacrylamide, and then visualization by Western blotting with distinct antibodies as indicated (Table S1). Some of the blotted membranes were stripped for $30 \mathrm{~min}$ and re-probed with additional primary antibodies. Therein, $\beta$-actin or GAPDH served as an internal control to verify equal loading of proteins.

\subsection{Subcutaneous tumor xenograft model}

Mouse xenograft models were made by subcutaneously heterotransplanting human HepG2 or derived $D D I 1^{-/}$cells. Briefly, equal amounts of cells $\left(1 \times 10^{7}\right)$ growing in the exponential phase were suspended in $0.1 \mathrm{ml}$ of serum-free medium and then inoculated subcutaneously at a single site in the right upper back region of male nude mice (BALB/C nu/nu, 4-6 weeks, $18 \mathrm{~g}$ ). The procedure of injection into all the mice was completed within $30 \mathrm{~min}$. Thereafter, the formation of murine subcutaneous tumor xenografts was successively observed until they were sacrificed. These transplanted tumors were excised immediately after being executed, and also calculated in size by a standard formulate $(\mathrm{V}=\mathrm{ab} 2 / 2)$. All mice were maintained under standard animal housing conditions with a 12-h dark cycle and also allowed access ad libitum to sterilized water and diet, according to the institutional guidelines for care and use of laboratory animals with a license SCXK (JING) 2019-0010. All experimental procedures were approved by the Ethics Committee of Chongqing Medical University.

\subsection{Pathohistology with H\&E staining}

The xenograft tumor tissues were immersed in 4\% paraformaldehyde overnight and then transferred to $70 \%$ ethanol. In processing cassettes, tumor tissues were dehydrated by a serial of alcohol gradient and then embedded in paraffin wax blocks, before being sectioned into a series of 5 - $\mu \mathrm{m}$-thick slides. Subsequently, the tissue sections were de-waxed in xylene, rehydrated through decreasing concentrations of ethanol and washed in PBS, before being stained by routine hematoxylin and eosin (H\&E) and visualized by microscopy. The resulting images were photographed herein.

\subsection{Statistical analysis}


bioRxiv preprint doi: https://doi.org/10.1101/2021.11.29.470338; this version posted November 29, 2021. The copyright holder for this preprint (which was not certified by peer review) is the author/funder. All rights reserved. No reuse allowed without permission.

Significant differences were statistically determined using Student's $t$-test and Multiple Analysis of Variations (MANOVA).

\section{Results and Discussion}

\subsection{Transcriptional expression of Nrf1 is unaffected by SREBP1.}

Based on the data obtained from chromatin immunoprecipitation (ChIP) (7) and microarray (29,30), Manning's group considered that aberrant activation of mTOR in $\mathrm{TSC2}^{-1-}$ MEFs led to upregulation of Nrf1 by SREBP1. However, we showed that transcriptional expression of Nrf1 was unaffected by SREBP1 knockdown or overexpression in both HepG2 and 7702 cell lines (Fig. 1, A to D). Almost no changes in transactivation of Nrf1 or Nrf2 promoter-driven luciferase reporters ( $p N r f 1$ luc and pNrf2-luc, established by Qiu et al (27)) were determined in the cellular response to rapamycin (RAPA), but they were induced by tert-butylhydroquinone (tBHQ, a pro-oxidative stressor) rather than the antioxidant N-Acetyl-L-cysteine (NAC) (Fig. 1, E to H). Such discrepancy may result from a strange disparity in the cytosolic to nuclear distribution of Nrf1 examined by Manning's lab (Fig. S1A, cropped from their extended data (7)). This is due to the authors showing abnormal accumulation of a peculiar nuclear Nrf1 isoform with a higher molecular weight than its cytosolic isoform, which is never recovered by all relevant subcellular fractionation experiments (cropped in Fig. S1B to D, as reported by our and other groups) (20,31-33). Such strange nuclear Nrf1 accumulation revealed by Manning's group seems to challenge against the well-established spatiotemporal character of this ER-localized transcription factor $(34,35)$.

Intriguingly, further examination showed that abundances of all Nrf1 isoforms were marginally enhanced by silencing of SREBP1 (Fig. 2A, cf. a2 vs a1). Accordantly increased mRNA levels of Nrf1-target PSMB6, PSMB7 and PSMB5 (encode the core proteasomal $ß 1, ß 2$ and $ß 5$ subunits, respectively (36)) were accompanied by enhanced protein abundances of PSMB6, PSMB7 and slightly PSMB5, concomitantly with SREBP1 knockdown (Fig. 2B,D). The difference between protein and mRNA levels of PSMB5 (as a key housekeeper in the ubiquitin-proteasomal system) suggests that its protein stability may be finely tuned by the proteasomal feedback regulatory loop, facilitating proteostasis maintenance. Conversely, phosphorylated S6 kinase 1 (pS6K1, required for protein synthesis) protein and mRNA expression levels were significantly down-regulated by SISREBP1 (Fig. 2B1, D). Thereby, SREBP1 may be directionally responsible for regulating protein synthesis and degradation.

Next, examination of SiSREBP1's effects on the upstream regulators of Nrf1 revealed that evident abundances of DDI1 and DDI2 were increased (Fig. 2C). This was accompanied by modest decreases in p97/VCP protein and mRNA levels, while the ER-resident E3 ligase Hrd3 was almost unaffected by silencing of SREBP1 (Fig. 2C, D). Further scrutinizing two distinct transcriptomic datasets (at https://www.ncbi.nIm.nih.gov/geo/query/acc.cgi?acc=GSE93980 and =GSE90571) unraveled that, though putative SREBP1-binding sites exist in both the promoter region of Nrf1 and its first exon (Fig. S2), no significant changes in transcriptional expression of Nrf1 and other homologous factors (Fig. 2E,F), as well as almost all proteasomal subunits (Fig. S3A,B), were determined in siSREBP1-expressing PANC-1 cells and SREBP1 ${ }^{-/}$MEFs, when compared to their wild-type controls. Collectively, these lines of evidence together demonstrate that Nrf1 is not a direct target of SREBP1, albeit its indirect effects on upstream signaling to Nrf1 cannot be ruled out.

\subsection{Discrete effects of rapamycin on the signaling to Nrf1 and proteasome.}

It was, to my surprise, found that stimulation of HepG2 cells by feeding $10 \%$ FBS after 10 -h free-serum starvation caused significant decreases in mRNA expression levels of Nrf1 and SREBP1, but their protein abundances were strikingly increased to varying extents (Fig. 3A vs B, C), and markedly diminished or abolished by rapamycin ( $20 \mathrm{nM}$, Fig. 3B, C). Similar results were obtained for S6K (Fig. 3C, D). Of note, mRNA expression of S6K, but not Nrf1 or SREBP1, was reversed and increased by rapamycin (Fig. 3D). Together, these demonstrate that mTOR is likely involved in at least two different mechanisms for regulating Nrf1 and SREBP1 at mRNA and protein expression levels, which are distinctive from controlling its downstream S6K1 by potential 'bounce-back' response to mTOR inhibitor.

Further experimental evidence showed that Nrf1-target PSMB5, PSMB6 and PSMB7 (Fig. 3B), as well as the upstream signaling DDI1, DDI2, p97 and Hrd1 (Fig. 3E) were significantly upregulated by feeding FBS, of which all those except Hrd3 were also inhibited by rapamycin. However, their mRNA expression levels were down-regulated or unaffected by FBS, but also partially reversed or event enhanced by rapamycin (Fig. 3F, cf. left vs right panels). Of note, mRNA expression of PSMB6 
and DDI2 was unaltered or down-regulated by FBS, respectively, but both were also significantly augmented by rapamycin. Overall, these results further indicate that key upstream and downstream signaling molecules of Nrf1 were, to some certain extents, influenced by mTOR involved in distinct hierarchical mechanisms.

\subsection{Alteration in the putative processing of Nrf1 in DDI1/2-deficient cells}

Since Nrf1 and C. elegans SKN-1A are activated by DDI1 in the proteasomal 'bounce-back' response $(23,26,28)$, we established a $D D / 1^{-/-}$cell line by CRISPR-editing with specific gRNA (Fig. S4A). Further examination of $D D / 1^{-/-}$cells by its DNA sequencing, real-time qPCR and Western blotting revealed that two overlapping nucleotide segments of $D D / 1$ were deleted from its two alleles (Figs. 4A \& S4B), but the remnant mRNA and protein levels were expressed (Fig.4, B and C). This implies there may exist alternative mRNA-splicing and in-frame translation start sites, as described in yeast DDI1 (37). In addition to $D D / 1^{-1}$, an extra-cytosine base was inserted in the open reading of $D D I 2$ (Fig. S4C), thus recalled $D D / 1^{-1-}\left(D D / 2^{i n s}\right)$. This should be a result of DDI1-recognized gRNA targeting the highly conserved sequence of DDI2 (Fig. S4A).

Assessment of subcutaneous tumor xenograft mice unraveled that no differences in in vivo tumorigenesis and tumor growth of $D D / 1^{-1-}\left(D D / 2^{i n s}\right)$ cells were observed when compared to those of its parent wild-type cells (Fig. 4, D and E). Also, no obvious changes in their tumor pathohistological sections were shown (in Fig. 4F). Such DDI1/2-deficient cells were then treated with distinct concentrations of MG132 and subjected to determination of putative effects of $D D / 1^{-/-}\left(D D / 2^{\text {ins }}\right)$ on the processing of Nrf1. As anticipated, the results revealed that processed Nrf1 isoforms-C/D were significantly reduced, but its full-length glycoprotein-A and deglycoprotein-B were almost unchanged following treatment of $D D / 1^{-/-}\left(D D / 2^{\text {ins } C}\right)$ with a lower dose $(1 \mu \mathrm{M})$ of MG132, when compared to those measured from wild-type cells (Fig. 4G, middle two lanes). By sharp contrast, a higher dose $(10 \mu \mathrm{M})$ of MG132 treatment of $D D / 1^{--}\left(D D / 2^{\text {ins }}\right)$ caused Nrf1 isoforms-C/D to be further diminished or abolished, but its full-length proteins-A/B were not augmented, when compared to their wild-type controls (Fig. 4G, right two lanes). These demonstrate a requirement of $26 \mathrm{~S}$ proteasome for DDI1/2-directed proteolytic processing of Nrf1, because stability of both proteases per se are also controlled by ubiquitin-proteasome pathways $(38,39)$. However, endogenous Nrf1 isoforms-A/B were marginally reduced in untreated $D D / 1^{-/}\left(D D I 2^{\text {ins }}\right)$ cells, where Nrf1 isoforms-C/D were rather faint to be distinguishable from wild-type controls (Fig. 4G, left two lanes). This implies that Nrf1 $\alpha$-derived isoforms may be much unstable to be rapidly destructed, but shorter isoforms Nrf1 ${ }^{\Delta N}, N r f 1 ß$ and Nrf1 $\gamma$ were unaffected, in DDI1/2deficient cells (Fig. 4G, left two lanes). Such seemingly-contradictory data, showing no increased full-length Nrf1 isoforms$\mathrm{A} / \mathrm{B}$ in $D D / 1^{-/-}\left(D D / 2^{\text {ins }}\right)$ cells, suggest that ER membrane-associated protein degradation and/or autophagy (40) may also be triggered in possibly 'bounce-back' response to $D D I 1 / 2$ deficiency.

Intriguingly, both wild-type DDI1 and its remnant protein in $D D I 1^{--}\left(D D I 2^{i n s}\right)$ cells were not enhanced, but modestly reduced by treatment of $1 \mu \mathrm{M}$ or $10 \mu \mathrm{M} \mathrm{MG132}$ for $4 \mathrm{~h}$ (Fig. $4 \mathrm{H}, h 1)$, and such reduced abundances were further decreased as treatment time was extended to $24 \mathrm{~h}$ (Fig. $4 \mathrm{H}, h 4)$, when compared with their untreated controls. By contrast, wild-type $\mathrm{DDI} 2$ and its remaining protein in $D D / 1^{-1}\left(D D / 2^{\text {ins }}\right)$ cells were largely unaffected by 24 -h treatment of $1 \mu \mathrm{M}$ or $10 \mu \mathrm{M} \mathrm{MG132}$ (Fig. $4 \mathrm{H}, h 5$ ), but after $4-\mathrm{h}$ treatment of cells, they became marginally reduced by $1 \mu \mathrm{M} M G 132$, and also rather augmented by $10 \mu \mathrm{M}$ MG132 (Fig. 4H, h2). Such distinct effects of this proteasomal inhibitor on DDI1 and DDI2 demonstrate that both protease stability may be governed through different mechanisms, albeit these details remain to be elucidated.

\subsection{DDI1/2-deficient effects on the rapamycin-responsive signaling to Nrf1.}

Herein, we also found marked decreases in the ectopic expression of Nrf1 $\alpha$-derived isoforms in $D D / 1^{-1-}\left(D D I 2^{\text {ins }}\right)$ cells, when compared to wild-type cells (Fig. 5A, $a 1$ and $a 3$ ). Further comparisons revealed that Nrf1-target proteasomal subunits PSMB5, PSMB6, PSMB7 were accordingly downregulated to considerably lower degrees in DDI1/2-deficient cells (Fig. 5A, a5 to a5). Also, $D D I 1 / 2$ deficiency enabled for significant downregulation of p97 (acting as a key upstream signaling to Nrf1) (Fig. 5B, b2), in addition to DDI2 and Nrf2 (Fig. 5B, b1 and b4). Rather, a relative increase in basal expression of Keap1 (as a negative regulator of $\mathrm{Nrf2}(41))$ was determined in $D D / 1^{-1-}\left(D D I 2^{i n s}\right)$ cells (Fig 5B, b5). Further real-time qPCR data unraveled various decreases in mRNA expression levels of $p 97, D D I 2, P S M B 5$, and PSMB7, but not of PSMB6, Nrf1 or Nrf2 in DDI1/2deficient cells (Fig. 5C). 
bioRxiv preprint doi: https://doi.org/10.1101/2021.11.29.470338; this version posted November 29, 2021. The copyright holder for this preprint (which was not certified by peer review) is the author/funder. All rights reserved. No reuse allowed without permission.

As such, abundances of endogenous Nrf1 $\alpha$-derived proteins were significantly increased by feeding $10 \%$ FBS for 12 $24 \mathrm{~h}$, after 10-h free-serum starvation of $D D / 1^{-1}\left(D D / 2^{\text {ins }}\right)$ cells, and these FBS-stimulated increases was almost completely abolished by $20 \mathrm{nM}$ rapamycin (Fig. 5D, d1). By contrast, FBS-stimulated protein expression of Nrf2 was partially suppressed by rapamycin (Fig. 5D, d2). However, mRNA expression levels of $N r f 1$ and $N r f 2$ were not induced, but rather repressed by FBS, and such repression was partially or completely reversed by rapamycin, as a result of enhanced mRNA expression of Nrf2 by this mTOR inhibitor (Fig. 5F and G). These data indicate there exists a feedback negative regulatory circuit between mRNA and protein expression of Nrf1 and Nrf2, during stimulation or inhibition of mTOR.

Further examinations of $D D I 1^{--}\left(D D I 2^{\text {ins }}\right)$ cells uncovered that the remnant $D D I 1$ and $D D I 2$ proteins were still partially enhanced by FBS, and also partially inhibited by rapamycin (Fig. 5E). Thereof, FBS-repressed mRNA expression of DDI2 was fully reversed to a slight increase by rapamycin (Fig. 5F, G). Similarly, modest stimulation of PSMB5, PSMB6 and PSMB7, abundances by FBS was also partially inhibited by rapamycin (Fig. $5 \mathrm{E}, e 3$ to e5), but their FBS-repressed mRNA levels were not obviously reversed by rapamycin (Fig. 5F,G). Such stimulatory or inhibitory effects of mTOR on rapamycin-responsive signaling to Nrf1 and downstream proteasome can partially occur in DDI1/2-deficient cells, implying a DDI1/2-independent mechanism also accounts for this process.

\subsection{Concluding remarks}

Since Nrf1 and SREBP1 manifest distinct topobiological behavior around membranes (42), they are thus endowed with distinctive spatiotemporal partitioning and unique biological functionality to be though exerted, only after being dislocated from the ER into nucleus so as to regulate distinct sets of target genes. In this study, we have discovered that Nrf1 is not a direct target of SREBP1, albeit both are involved in rapamycin-responsive signaling networks (Fig. 6). Of note, the upstream signaling to Nrf1 dislocation by p97 and its processing by DDI1/2, along with downstream proteasomal expression, should be indirectly monitored by potent mTOR signaling networks, to various certain extents, depending on distinct experimental settings in distinct cell types. Thereof, potential indirect effects of SREBP1 on DDI1/2 and proteasomes cannot be ruled out. Further experimental evidence from $D D I 1^{--}\left(D D I 2^{\text {ins }}\right)$ cells demonstrates that putative effects of mTOR on the rapamycinresponsive signaling to $\mathrm{Nrf1}$ and proteasome may also be executed partially through a DDI1/2-independent mechanism, albeit the detailed regulatory events remain to be elucidated.

Author contributions: K.L., S.H., and L.Q. performed all the experiments with help of M.W., collected all the relevant data, and made the manuscript draft with figures and supplemental information. M.W. did bioinformatics analysis of datasets. As a native English-speaker, Z.Z. helped to polish the English language and edited this manuscript. Lastly, Y.Z. designed and supervised this study, analyzed all the data, helped to prepare all figures with cartoons, wrote and revised the paper.

Acknowledgments: We thank to all those present and past members of Prof. Zhang's laboratory (at Chongqing University, China) for giving critical discussion and invaluable help with this work. Notably, this study was also funded by the National Natural Science Foundation of China (NSFC, with two project grants 81872336 and 82073079 ) awarded to Prof. Yiguo Zhang.

Conflicts of Interest: The authors declare no conflict of interest.

\section{References}

1. Lopez-Otin, C., and Kroemer, G. (2021) Hallmarks of health. Cell 184, 1929-1939

2. Hanahan, D., and Weinberg, R. A. (2011) Hallmarks of cancer: the next generation. Cell 144, 646-674

3. Lopez-Otin, C., Blasco, M. A., Partridge, L., Serrano, M., and Kroemer, G. (2013) The hallmarks of aging. Cell 153, 1194-1217

4. Kotas, M. E., and Medzhitov, R. (2015) Homeostasis, inflammation, and disease susceptibility. Cell 160, 816-827

5. Osinalde, N., Duarri, A., Ramirez, J., Barrio, R., Perez de Nanclares, G., and Mayor, U. (2019) Impaired proteostasis in rare neurological diseases. Semin Cell Dev Biol 93, 164-177

6. Toyama, B. H., and Hetzer, M. W. (2013) Protein homeostasis: live long, won't prosper. Nat Rev Mol Cell Biol 14, 55-61

7. Zhang, Y., Nicholatos, J., Dreier, J. R., Ricoult, S. J., Widenmaier, S. B., Hotamisligil, G. S., Kwiatkowski, D. J., and Manning, B. D. (2014) Coordinated regulation of protein synthesis and degradation by mTORC1. Nature 513, 440-443

8. Aramburu, J., Ortells, M. C., Tejedor, S., Buxade, M., and Lopez-Rodriguez, C. (2014) Transcriptional regulation of the stress response by mTOR. Sci Signal 7, re2

9. Zhang, Y., and Manning, B. D. (2015) mTORC1 signaling activates NRF1 to increase cellular proteasome levels. Cell Cycle 14, 2011-2017

10. Laplante, M., and Sabatini, D. M. (2009) An emerging role of mTOR in lipid biosynthesis. Curr Biol 19, R1046-1052 
bioRxiv preprint doi: https://doi.org/10.1101/2021.11.29.470338; this version posted November 29, 2021. The copyright holder for this preprint (which was not certified by peer review) is the author/funder. All rights reserved. No reuse allowed without permission.

11. Sakai, J., and Rawson, R. B. (2001) The sterol regulatory element-binding protein pathway: control of lipid homeostasis through regulated intracellular transport. Curr Opin Lipidol 12, 261-266

12. Wang, B., and Tontonoz, P. (2018) Liver $X$ receptors in lipid signalling and membrane homeostasis. Nat Rev Endocrinol

13. Zhao, J., Garcia, G. A., and Goldberg, A. L. (2016) Control of proteasomal proteolysis by mTOR. Nature 529, E1-2

14. He, C., and Klionsky, D. J. (2009) Regulation mechanisms and signaling pathways of autophagy. Annu Rev Genet 43, 67-93

15. Zhao, J., Zhai, B., Gygi, S. P., and Goldberg, A. L. (2015) mTOR inhibition activates overall protein degradation by the ubiquitin proteasome system as well as by autophagy. Proc Natl Acad Sci U S A 112, 15790-15797

16. Saxton, R. A., and Sabatini, D. M. (2017) mTOR Signaling in Growth, Metabolism, and Disease. Cell 168, 960-976

17. Zhang, Y., and Manning, B. D. (2016) Zhang \& Manning reply. Nature 529, E2-3

18. Shimobayashi, M., and Hall, M. N. (2014) Making new contacts: the mTOR network in metabolism and signalling crosstalk. Nat Rev Mol Cell Biol 15, 155-162

19. Zheng, Z., Wang, M., Hu, S., Deng, R., Feng, J., and Zhang, Y. (2021) The inflammatory accumulation of lipids and reactive oxygen species in human Nrf1 $\alpha$-deficient hepatoma cells is ameliorated by 2-bromopalmitate. BioRxiv doi: https://doi.org/ 10.1101/2021.09.29.462358

20. Widenmaier, S. B., Snyder, N. A., Nguyen, T. B., Arduini, A., Lee, G. Y., Arruda, A. P., Saksi, J., Bartelt, A., and Hotamisligil, G. S. (2017) NRF1 Is an ER Membrane Sensor that Is Central to Cholesterol Homeostasis. Cell 171, 1094-1109 e1015

21. Liu, F., Ma, M., Gao, A., Ma, F., Ma, G., Liu, P., Jia, C., Wang, Y., Donahue, K., Zhang, S., Ong, I. M., Keles, S., Li, L., and Xu, W. (2021) PKM2-TMEM33 axis regulates lipid homeostasis in cancer cells by controlling SCAP stability. EMBO J, e108065

22. Sirkis, R., Gerst, J. E., and Fass, D. (2006) Ddi1, a eukaryotic protein with the retroviral protease fold. J Mol Bio/ 364, 376-387

23. Lehrbach, N. J., and Ruvkun, G. (2016) Proteasome dysfunction triggers activation of SKN-1A/Nrf1 by the aspartic protease DDI-1. Elife 5

24. Motyan, J. A., Miczi, M., and Tozser, J. (2020) Dimer Interface Organization is a Main Determinant of Intermonomeric Interactions and Correlates with Evolutionary Relationships of Retroviral and Retroviral-Like Ddi1 and Ddi2 Proteases. Int J Mol Sci 21

25. Siva, M., Svoboda, M., Veverka, V., Trempe, J. F., Hofmann, K., Kozisek, M., Hexnerova, R., Sedlak, F., Belza, J., Brynda, J., Sacha, P., Hubalek, M., Starkova, J., Flaisigova, I., Konvalinka, J., and Saskova, K. G. (2016) Human DNA-Damage-Inducible 2 Protein Is Structurally and Functionally Distinct from Its Yeast Ortholog. Sci Rep 6, 30443

26. Xiang, Y., Halin, J., Fan, Z., Hu, S., Wang, M., Qiu, L., Zhang, Z., Mattjus, P., and Zhang, Y. (2018) Topovectorial mechanisms control the juxtamembrane proteolytic processing of $\mathrm{Nrf1}$ to remove its $\mathrm{N}$-terminal polypeptides during maturation of the CNC-bZIP factor. Toxicol Appl Pharmacol 360, 160-184

27. Qiu, L., Wang, M., Hu, S. F., Ru, X. F., Ren, Y. G., Zhang, Z. W., Yu, S. W., and Zhang, Y. G. (2018) Oncogenic Activation of Nrf2, Though as a Master Antioxidant Transcription Factor, Liberated by Specific Knockout of the Full-Length Nrf1 alpha that Acts as a Dominant Tumor Repressor. Cancers 10

28. Xiang, Y., Wang, M., Hu, S., Qiu, L., Yang, F., Zhang, Z., Yu, S., Pi, J., and Zhang, Y. (2018) Mechanisms controlling the multistage post-translational processing of endogenous Nrf1alpha/TCF11 proteins to yield distinct isoforms within the coupled positive and negative feedback circuits. Toxicol Appl Pharmacol 360, 212-235

29. Rome, S., Lecomte, V., Meugnier, E., Rieusset, J., Debard, C., Euthine, V., Vidal, H., and Lefai, E. (2008) Microarray analyses of SREBP-1a and SREBP-1c target genes identify new regulatory pathways in muscle. Physiol Genomics 34, 327-337

30. Reed, B. D., Charos, A. E., Szekely, A. M., Weissman, S. M., and Snyder, M. (2008) Genome-wide occupancy of SREBP1 and its partners NFY and SP1 reveals novel functional roles and combinatorial regulation of distinct classes of genes. PLoS Genet 4, e1000133

31. Sotzny, F., Schormann, E., Kuhlewindt, I., Koch, A., Brehm, A., Goldbach-Mansky, R., Gilling, K. E., and Kruger, E. (2016) TCF11/Nrf1-Mediated Induction of Proteasome Expression Prevents Cytotoxicity by Rotenone. Antioxid Redox Signal 25, 870885

32. Wang, M., Ren, Y., Hu, S., Liu, K., Qiu, L., and Zhang, Y. (2021) TCF11 Has a Potent Tumor-Repressing Effect Than Its Prototypic Nrf1alpha by Definition of Both Similar Yet Different Regulatory Profiles, With a Striking Disparity From Nrf2. Front Oncol 11, 707032

33. Zhang, Y., Lucocq, J. M., and Hayes, J. D. (2009) The Nrf1 CNC/bZIP protein is a nuclear envelope-bound transcription factor that is activated by $\mathrm{t}$-butyl hydroquinone but not by endoplasmic reticulum stressors. Biochem J 418, 293-310

34. Zhang, Y., and Xiang, Y. (2016) Molecular and cellular basis for the unique functioning of Nrf1, an indispensable transcription factor for maintaining cell homoeostasis and organ integrity. Biochem $J \mathbf{4 7 3}, 961-1000$

35. Yuan, J., Zhang, S., and Zhang, Y. (2018) Nrf1 is paved as a new strategic avenue to prevent and treat cancer, neurodegenerative and other diseases. Toxicol Appl Pharmacol 360, 273-283

36. Mao, Y. (2021) Structure, Dynamics and Function of the 26S Proteasome. Subcell Biochem 96, 1-151

37. Liu, Y., Dai, H., and Xiao, W. (1997) UAS(MAG1), a yeast cis-acting element that regulates the expression of MAG1, is located within the protein coding region of DDI1. Mol Gen Genet 255, 533-542

38. Yip, M. C. J., Bodnar, N. O., and Rapoport, T. A. (2020) Ddi1 is a ubiquitin-dependent protease. Proc Natl Acad Sci U S A 117, 7776-7781

39. Dirac-Svejstrup, A. B., Walker, J., Faull, P., Encheva, V., Akimov, V., Puglia, M., Perkins, D., Kumper, S., Hunjan, S. S., Blagoev, B., Snijders, A. P., Powell, D. J., and Svejstrup, J. Q. (2020) DDI2 Is a Ubiquitin-Directed Endoprotease Responsible for Cleavage 
of Transcription Factor NRF1. Mol Cell 79, 332-341 e337

40. Wang, X. J., Yu, J., Wong, S. H., Cheng, A. S., Chan, F. K., Ng, S. S., Cho, C. H., Sung, J. J., and Wu, W. K. (2013) A novel crosstalk between two major protein degradation systems: regulation of proteasomal activity by autophagy. Autophagy $\mathbf{9}, 1500-1508$

41. Baird, L., and Yamamoto, M. (2020) The Molecular Mechanisms Regulating the KEAP1-NRF2 Pathway. Mol Cell Biol 40

42. Zhu, Y. P., Wang, M., Xiang, Y., Qiu, L., Hu, S., Zhang, Z., Mattjus, P., Zhu, X., and Zhang, Y. (2018) Nach Is a Novel Subgroup at an Early Evolutionary Stage of the CNC-bZIP Subfamily Transcription Factors from the Marine Bacteria to Humans. Int J Mol Sci 19, 1-26

Figure legends
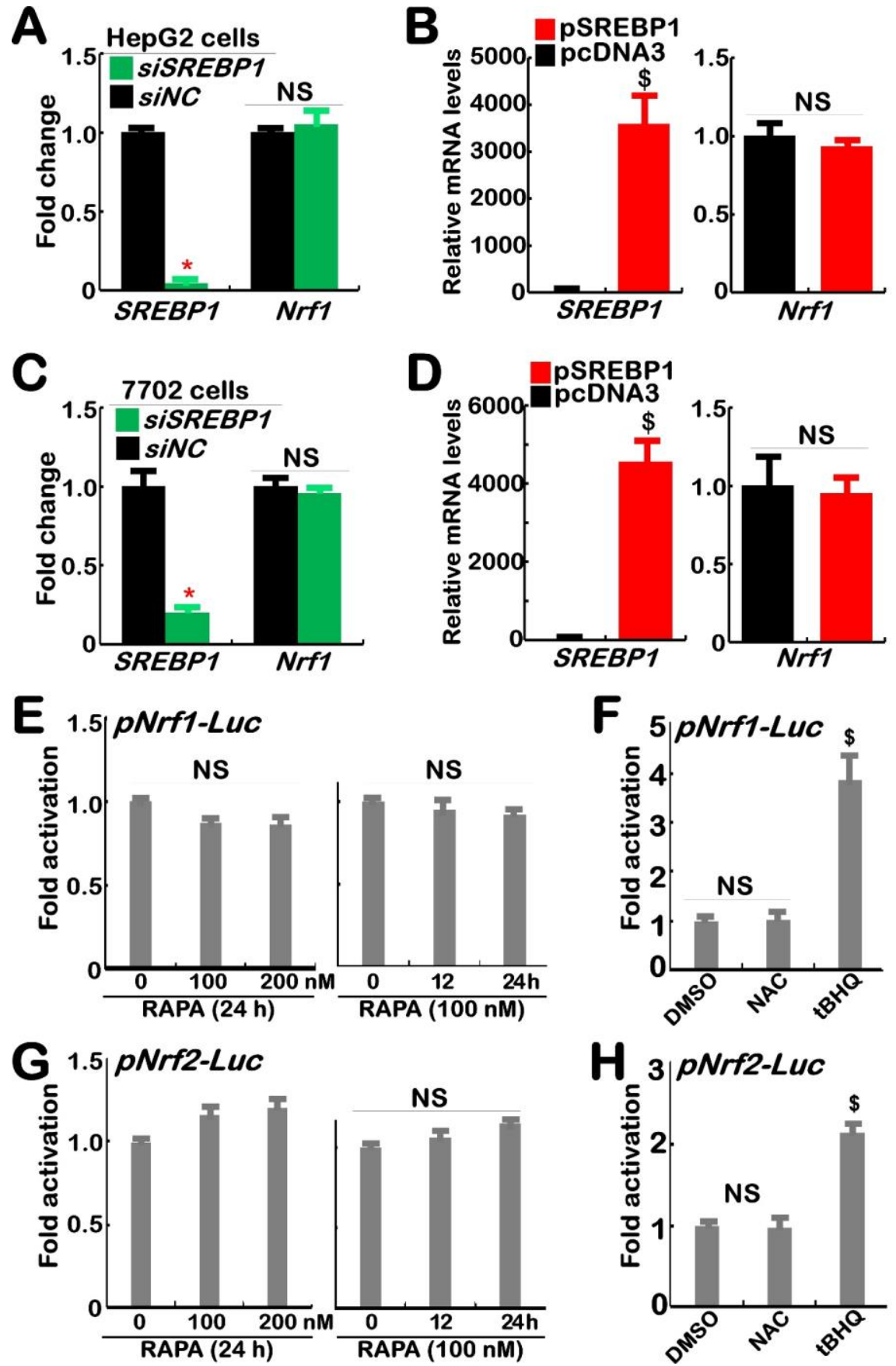

Figure 1. Transcriptional expression of Nrf1 and its reporter is unaffected by SREBP1 or rapamycin.

(A to $D)$ Two cell lines of HepG2 $(A, B)$ and $L 7702(C, D)$, that had been transfected with: $(A, C)$ siNC (a negative control) or siSREBP1; $(B, D)$ a pSREBP1 expression construct or empty plasmid, were subjected to real-time qPCR analysis of mRNA expression levels of SREBP1 and Nrf1 ( $\mathrm{n}=3 \times 3$; with significant decreases $(*, p<0.01)$, significant increases $(\$, p<0.01)$, or no significances (NS)). (E to H) HepG2 cells, that had been transfected with $p N r f 1-I u c(E, F)$ or $p N r f 2-l u c(G, H)$ reporters, along with $p R L-T K$ (an internal control) and then treated for $24 \mathrm{~h}$ with rapamycin (RAPA, at 0,100 or $200 \mathrm{nM})(E, G), \mathrm{NAC}(10 \mathrm{mM})$ or tBHQ $(50 \mu \mathrm{M})(F, H)$, were subjected to assay of dual luciferase activity $(n=3 \times 3)$ with significant increases $(\$, p<0.01)$ or no significances (NS). All the results representing at least three independent experiments, each of which was performed in triplicates, were determined as fold changes (mean \pm S.D.) relative to equivalent controls. 

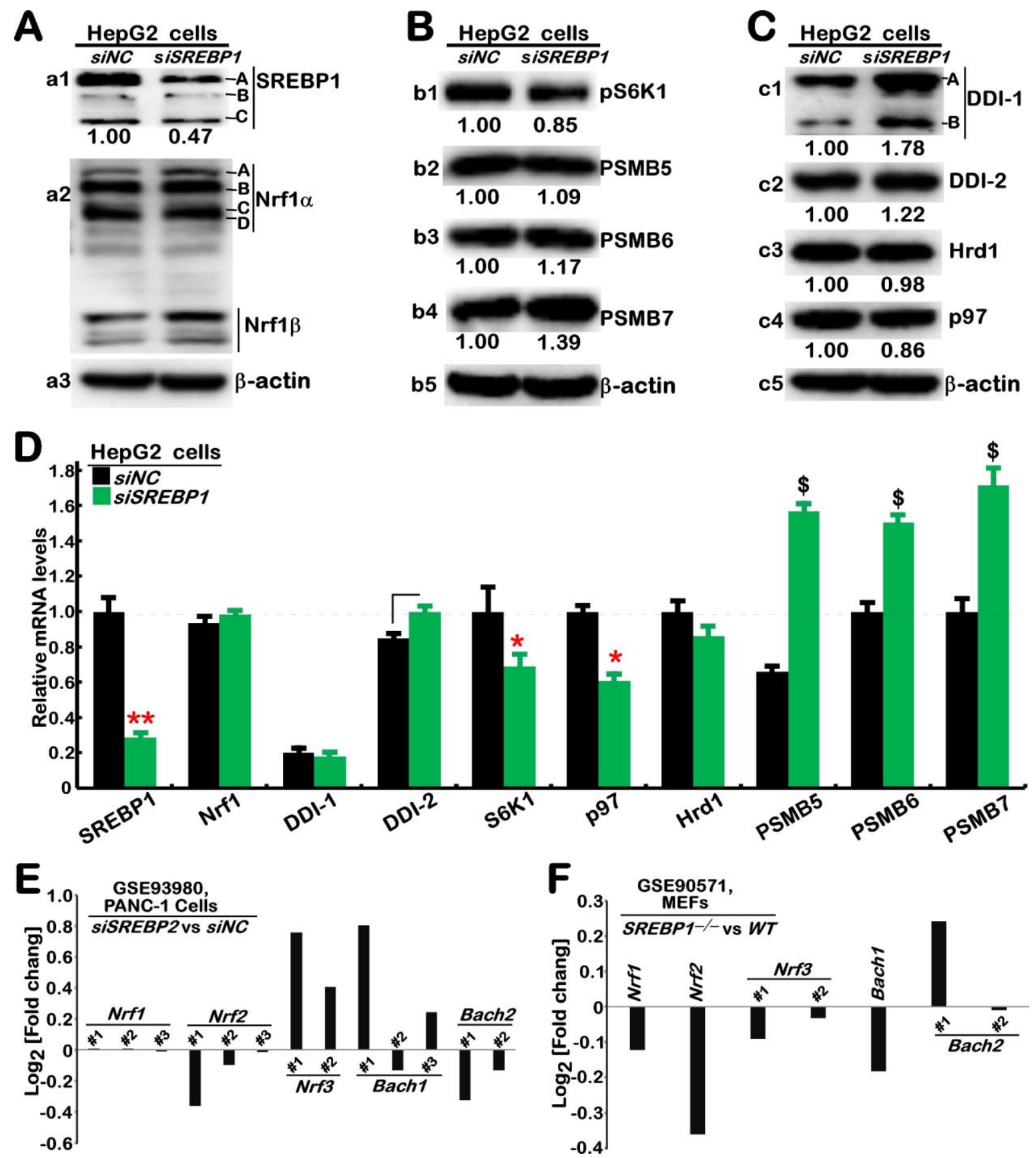

Figure 2. The upstream signaling to Nrf1 and proteasome are to no or less degrees, affected in SREBP1-deficient cells.

(A to C) HepG2 cells were transfected with siNC or siSREBP1 for $24 \mathrm{~h}$ and then subjected to Western blotting with those indicated antibodies. The intensity of immunoblots representing each protein was quantified by the Quantity-One software and shown on the bottom. (D) The mRNA levels of those examined genes were determined by real-time qPCR and shown as fold changes (mean \pm S.D. $n=3 \times 3)$ with significant decreases $(*, p<0.01)$ or significant increases $(\$, p<0.01)$ relative to equivalent controls. $(E, F)$ No significant changes in transcriptional expression of $\mathrm{Nrf1}$ and other homologous factors were determined by transcriptomic sequencing of siSREBP2 vs siNC (https://www.ncbi.nlm.nih.gov/geo/query/acc.cgi?acc=GSE93980) in PANC-1 cells (E) or SREBP1- vs Wild-type MEFs ( https://www.ncbi.nlm.nih.gov/geo/query/acc.cgi?acc=GSE90571) (F). 

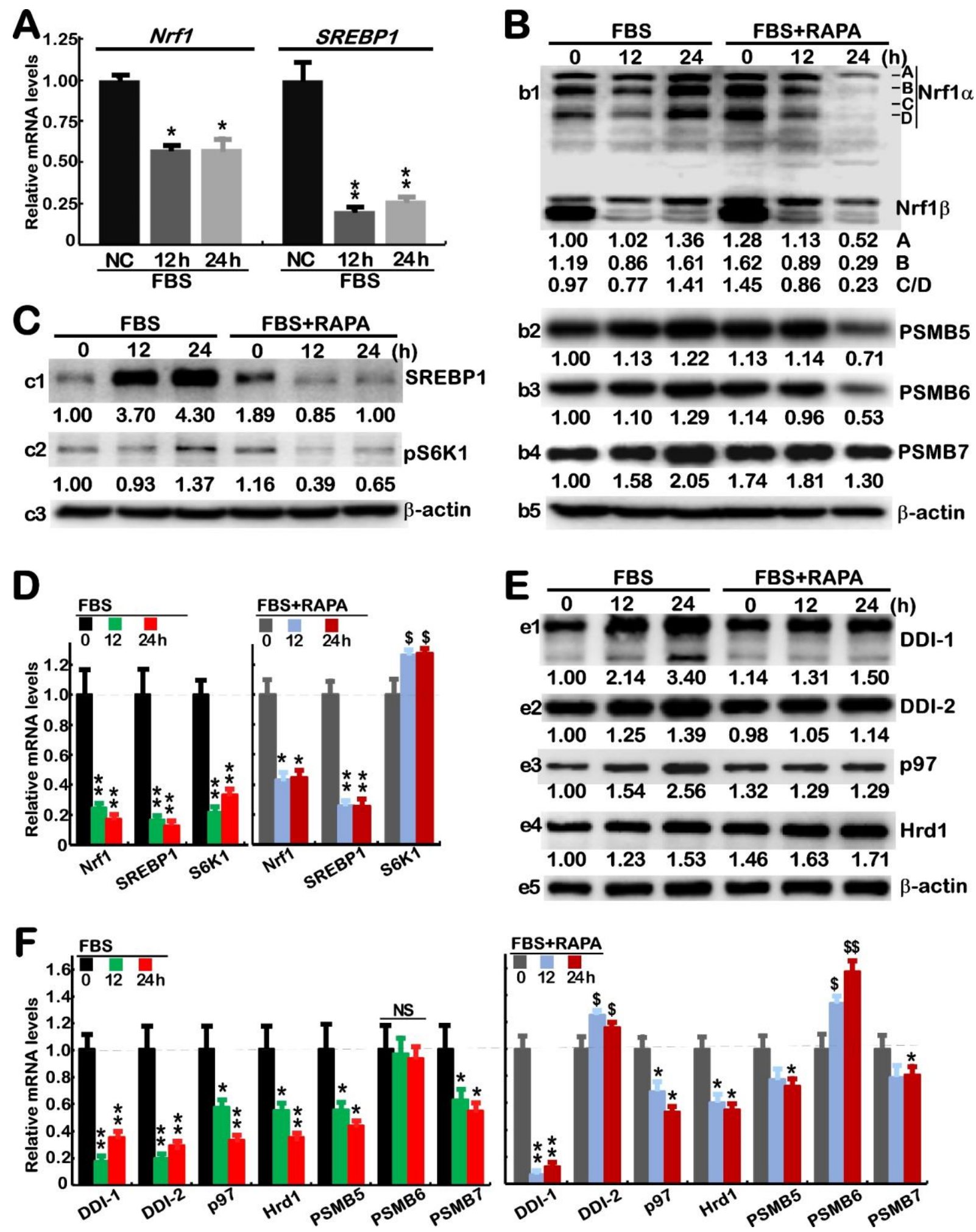

Figure 3. Distinct effects of rapamycin on FBS-altered expression of SREBP1, Nrf1 and relevant signaling molecules.

(A) HepG2 cells that had been starved in a serum-free medium for $10 \mathrm{~h}$ and then stimulated for 12 or $24 \mathrm{~h}$ by feeding $10 \% \mathrm{FBS}$, were subjected to real-time qPCR analysis of Nrf1 and SREBP1 at mRNA expression levels. The results were shown as fold changes (mean \pm S.D. $\mathrm{n}=3 \times 3$ ) with significant decreases $(*, p<0.01 ; * *, p<0.001$ ) relative to the negative controls (NC, with no FBS treatment). (B to F) The free-serum starved HepG2 cells were treated with 10\% FBS alone or plus 20 nM RAPA for $0,12,24 \mathrm{~h}$, before being subjected to Western blotting with indicated antibodies $(B, C, E)$, in which the intensity of immunoblots was calculated and shown on the bottom, or real-time qPCR analysis of indicated genes at mRNA levels $(D, F)$. The results were shown as fold changes (mean \pm S.D. $\mathrm{n}=3 \times 3$ ) with significant decreases $\left(*, p<0.01 ;{ }^{* *}, p<0.001\right)$, significant increases $(\$, p<0.01 ; \$ \$$, $p<0.001$ ), or no significant differences (NS), relative to their equivalent controls. 


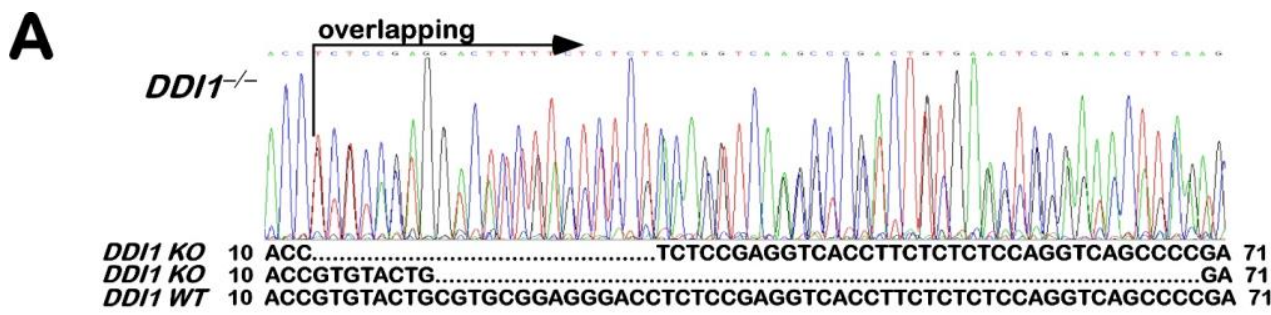

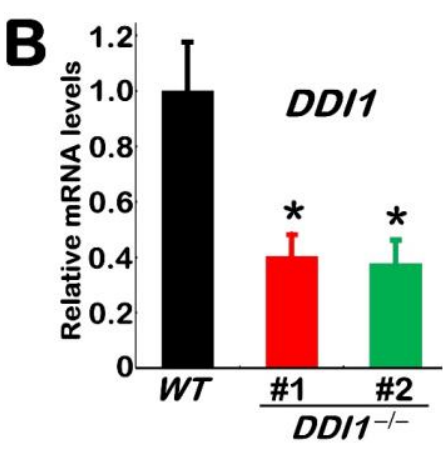

$\mathbf{E}$

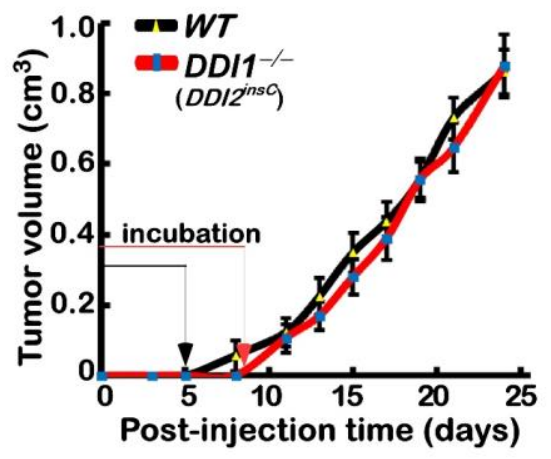

G

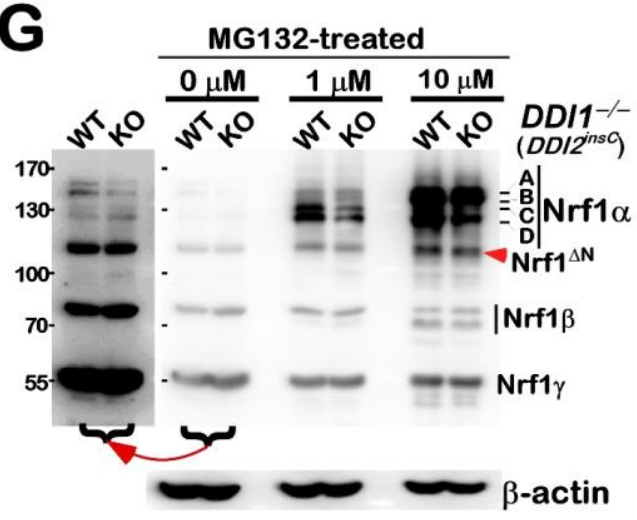

C

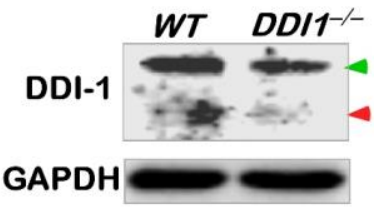

$\mathbf{F}$

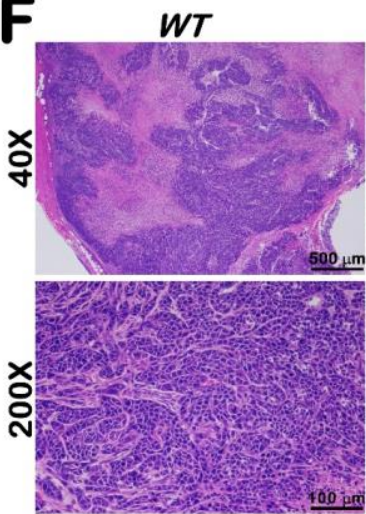

D

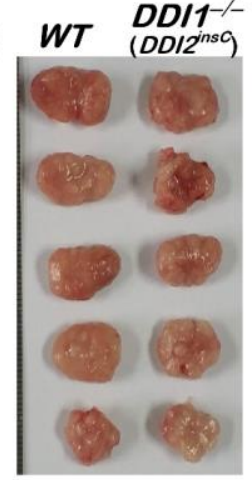

$D D 11^{-1-}\left(D D / 2^{\text {ns }}\right)$

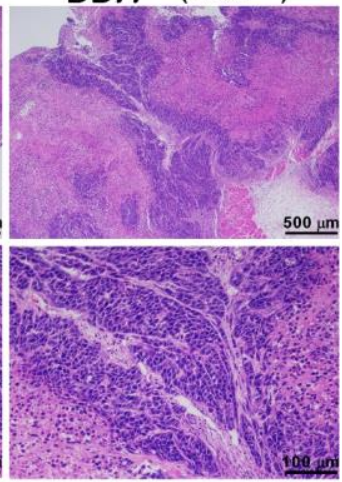

Figure 4. Changed processing of Nrf1 in DDI1/2-deficient cells, but with no different xenograft models.

(A) HepG2-derived $D D I 1^{-1-}$ cells were initially identified by its genomic DNA-sequencing. The results were shown graphically, along with alignment of two mutant alleles and wild-type $(W T)$. (B, C) In contrast with $W T$ cells, $D D / 1^{-1}$ cells were further determined by real-time $q P C R\left(B\right.$, shown by mean \pm S.D. $\left.n=3 \times 3 ;{ }^{*}, p<0.01\right)$ and Western blotting $(C)$, respectively. (D) No different phenotypes of xenograft tumors in nude mice were observed after murine subcutaneous inoculation of $W T$ and $D D I 1^{-1-}\left(D D I 2^{\text {ins } C}\right)$ hepatoma cells. (D) No differences in both tumorigenesis and in vivo growth of between WT and DDI1/2-deficient and xenograft tumors were measured in size every two days, before being sacrificed. The results are shown as mean $\pm \operatorname{SEM}(n=5)$. (F) The pathohistological images were obtained by routine HE staining of aforementioned xenograft tumor tissues. (G, h) Both lines of WT and KO (i.e. DDI1 ${ }^{--} D D I 2^{\text {ins }}$ ) cells were treated with MG132 at 0,1 or $10 \mu \mathrm{M}$ for $24 \mathrm{~h}(G, H)$ or $4 \mathrm{~h}(H)$, and then subjected to Western blotting with distinct antibodies against Nrf1, DDI1 or DDI2. In addition, a long-term exposed image was cropped from part of the corresponding gel $(G)$. 

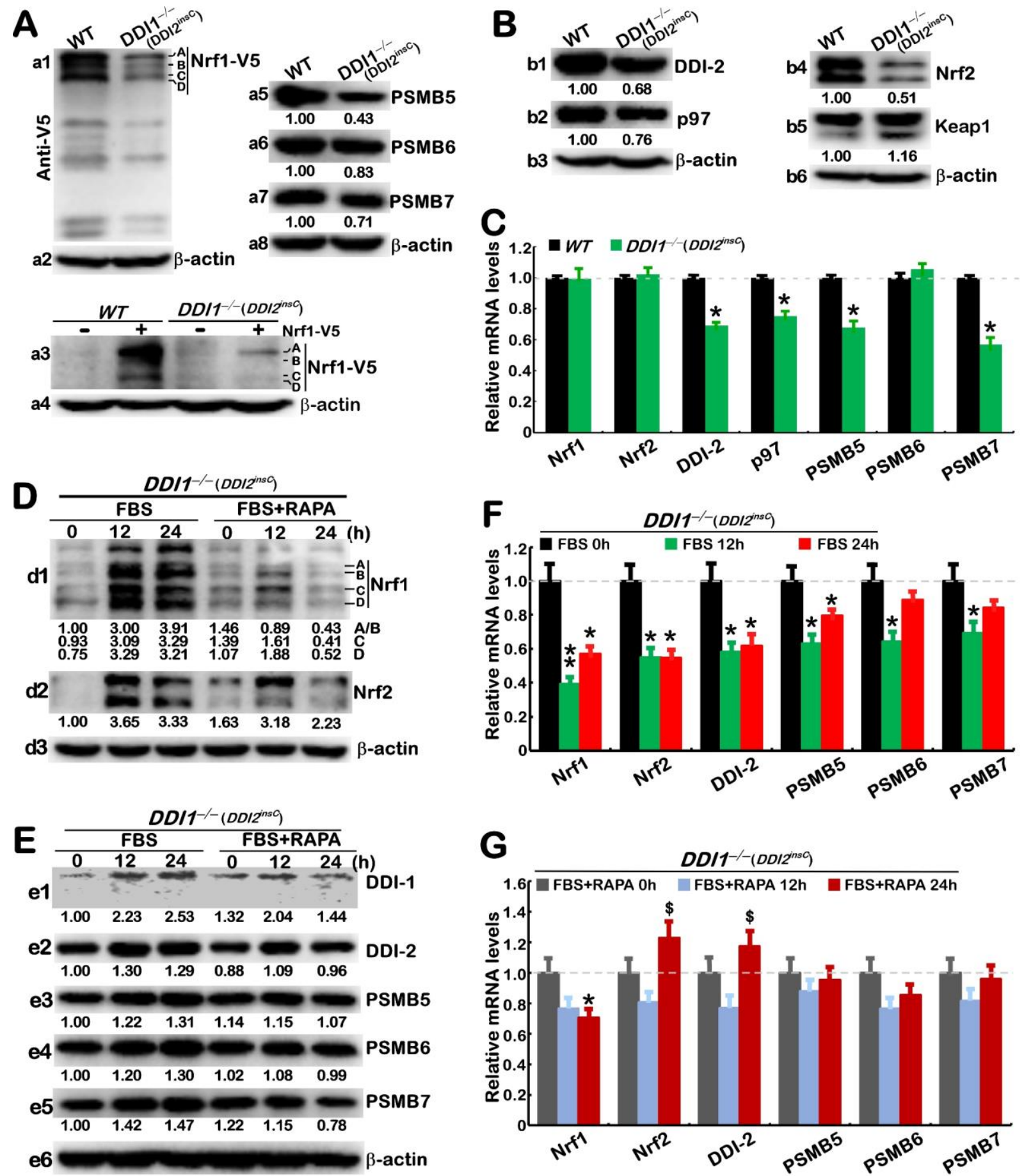

Figure 5. DDI1/2-deficient effects on the rapamycin-responsive signaling to Nrf1 and proteasome.

(A) $W T$ and $D D I 1^{-/-}\left(D D I 2^{\text {insC }}\right)$ cell lines were transfected with an expression construct for Nrf1-V5 (+) or empty pcDNA3 vector (-) and then examined by Western blotting with V5 antibody $(a 1, a 3)$; ß-actin acts as a loading control. Their untransfected cells were also measured by immunoblotting of the core proteasomal subunits PSMB5, PSMB6, and PSMB7 (a5 to a7). (B) Further immunoblotting of DDI2, p97, Nrf2 and Keap1 was conducted in untreated $W T$ and $D D I 1^{-1-}\left(D D I 2^{\text {ins }}\right)$ cell lines. (C) Both cell lines were further assessed by real-time qPCR analysis of mRNA expression levels. The results were shown as fold changes (mean \pm S.D. $\mathrm{n}=3 \times 3$ ) with significant decrease $(*, p<0.01)$ relative to control values. ( $D$ to $\mathrm{G})$ The starved $D D / 1^{-/-}\left(D D I 2^{\text {ins } C}\right)$ cells were treated by feeding $10 \%$ FBS alone or plus RAPA ( $20 \mathrm{nM}$ ) for 0,12 or $24 \mathrm{~h}$, and then subjected to Western blotting with distinct antibodies ( $D$, $E)$ and real-time $q P C R$ analysis of mRNA expression $(F, G)$. The resulting data were shown as fold changes (mean $\pm S . D . n=3 \times 3$ ), with significant decrease $(*, p<0.01)$ or significant increases $(\$, p<0.01)$ relative to control values. 


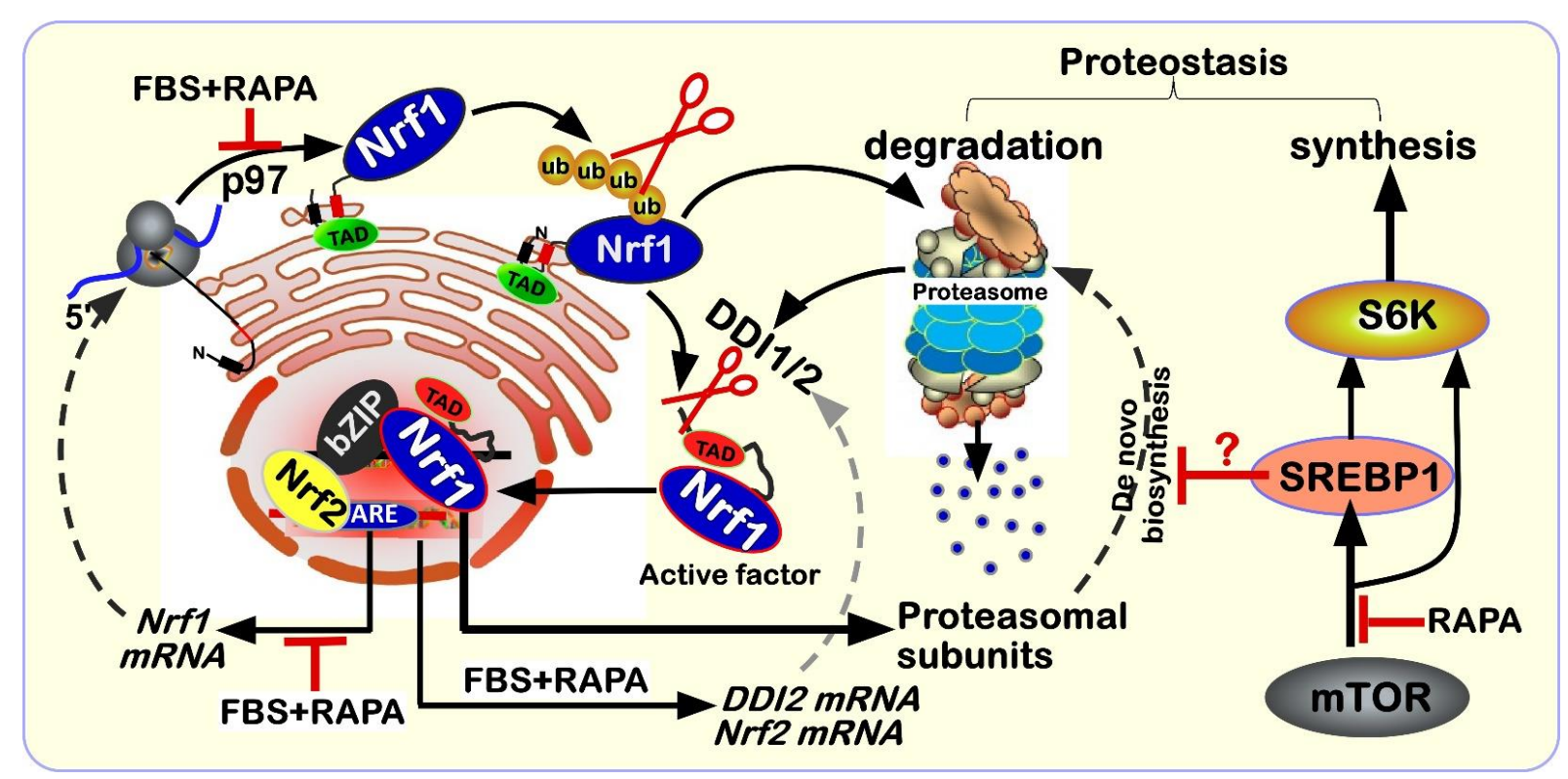

Figure 6. A model is proposed for a better understanding of the rapamycin-responsive signaling to Nrf1 and proteasomes.

The ER-localized Nrf1 manifests its unique topobiological behavior with specific dislocation by p97 and proteolytic processing by DDI1/2 and proteasomes, to give rise to a mature N-terminally-truncated isoform of this CNC-bZIP factor that mediates proteasomal transcriptional expression. Herein, we found that Nrf1 is not a direct target of SREBP1 (required for lipid and cholesterol metabolism) and Nrf1-target proteasomal transcription is almost not induced, but rather inhibited by SREBP1, although both factors are also integrated into the rapamycin-responsive signaling networks. Besides, differential expression levels of p97, DDI1/2 and Nrf2 may also be monitored by mTOR signaling, to various certain extents, depending on distinct experimental setting in distinct cell types. These detailed regulatory mechanisms should warrant for in-depth studies. 